\title{
Research
}

\section{Antibiotic prescribing quality for children in primary care:}

\author{
an observational study
}

\begin{abstract}
Background

Overuse and inappropriate prescribing of antibiotics is driving antibiotic resistance. GPs often prescribe antibiotics for upper respiratory tract infections (URTIs) in young children despite their marginal beneficial effects.

\section{Aim}

To assess the quality of antibiotic prescribing for common infections in young children attending primary care and to investigate influencing factors.
\end{abstract}

\section{Design and setting}

An observational, descriptive analysis, including children attending primary care sites in England and Wales.

\section{Method}

The Diagnosis of Urinary Tract infection in Young children study collected data on 7163 children aged $<5$ years, presenting to UK primary care with an acute illness ( $<28$ days). Data were compared with the European Surveillance of Antimicrobial Consumption Network (ESACNet) disease-specific quality indicators to assess prescribing for URTIs, tonsillitis, and otitis media, against ESAC-Net proposed standards. Non-parametric trend tests and $X^{2}$ tests assessed trends and differences in prescribing by level of deprivation, site type, and demographics

\section{Results}

Prescribing rates fell within the recommendations for URTIs but exceeded the recommended limits for tonsillitis and otitis media. The proportion of children receiving the recommended antibiotic was below standards for URTIs and tonsillitis, but within the recommended limits for otitis media. Prescribing rates increased as the level of deprivation decreased for all infections $(P<0.05)$ and increased as the age of the child increased for URTIs and tonsillitis ( $P<0.05)$. There were no other significant trends or differences.

\section{Conclusion}

The quality of antibiotic prescribing in this study was mixed and highlights the scope for future improvements. There is a need to assess further the quality of disease-specific antibiotic prescribing in UK primary care settings using data representative of routine clinical practice.

\section{Keywords}

antibiotic prescribing; children; ESAC-Net disease-specific quality indicators; primary care; respiratory tract infections.
BACKGROUND

Antibiotic resistance is an important public health concern. ${ }^{1,2}$ The number of infections caused by resistant bacteria are increasing and there are limited new replacement classes of antibiotics.3,4 Overuse and inappropriate prescribing of antibiotics is driving antibiotic resistance. Acute illness in young children is one of the commonest reasons for patients to seek health care worldwide and respiratory tract infections are responsible for $74.4 \%$ of antibiotic prescriptions in children, ${ }^{5}$ despite their marginal beneficial effects. ${ }^{6}$ Goodquality antibiotic prescribing for children in primary care is key to controlling antibiotic resistance. European quality indicators for antibiotic prescribing have been developed for primary care, but the extent to which these are achieved in the UK is unknown.?

There is a need to assess the standards of antibiotic prescribing in primary care, especially as $90 \%$ of antibiotics are prescribed by GPs. ${ }^{8}$ In the UK, antibiotics are still being overprescribed despite published guidelines.? Levels of GP antibiotic prescribing has increased by 40\% between 1999 and 2011, and by 4.1\% between 2010 and 2013.10 Evidence shows a strong association between antibiotic exposure and antibiotic resistance. ${ }^{11}$ Poor antibiotic prescribing exposes patients to unnecessary drug treatment and leads to

MR Williams, BSc, medical student, School of Medicine, Cardiff University, Cardiff. G Greene, PhD, lecturer; G Naik, MS(surgery), MRCSEd, MRCGP, MPH, GP, honorary research fellow, Department of Population Medicine, Cardiff University, Cardiff. K Hughes, MRCGP, PhD, DCH, clinical research fellow, PRIME Centre Wales, Department of Population Medicine, Cardiff University, Cardiff. CC Butler, MD, FRCGP, FFPH, FMedSci, DCH, CCH, GP, professor of primary care, Nuffield Department of Primary Care Health Sciences, University of Oxford, Oxford. AD Hay, MD, MRCP, FRCGP, DCH, GP, professor of primary care and NIHR Research Professor, Centre for Academic Primary Care, Population increased costs to the health service and higher morbidity and mortality rates, as a result of infections caused by resistant bacteria. ${ }^{1,12}$

Diagnostic complexity ${ }^{13}$ and prognostic uncertainty $^{14}$ play important roles in decisions regarding antibiotic prescriptions, and GPs are more likely to prescribe if they perceive pressure from parents. ${ }^{15}$ Parental anxiety and pressures of time influence prescribing decisions, and delayed antibiotic prescribing is used to increase parental confidence and maintain good relationships with parents. ${ }^{16}$

In order to improve antibiotic prescribing, it is important to measure its quality. The European Surveillance of Antimicrobial Consumption Network (ESAC-Net) (previously European Surveillance of Antimicrobial Consumption [ESAC]] developed and validated disease-specific antibiotic prescribing quality indicators. This group consisted of 40 experts from 25 different countries. There was consensus that these quality indicators can be used to assess the quality of antibiotic prescribing and, therefore, can be used to improve it.? Several studies have assessed the relationship between the ESAC-Net indicators and prescribing behaviours., $517-19$ However, none has examined this in a large cohort of young children in the UK.

The aim of this project was to assess

Health Sciences, Bristol Medical School, Bristol. Address for correspondence

Giles Greene, Cardiff University, Division of

Population Medicine, Neuadd Meirionnydd, Heath Park, Cardiff CF14 4XN, UK

E-mail: GreeneGAcf.ac.uk

Submitted: 15 May 2017; Editor's response: 4 July 2017; final acceptance: 13 September 2017. CBritish Journal of General Practice

This is the full-length article (published online 16 Jan 2018) of an abridged version published in print. Cite this version as: Br J Gen Pract 2018; DOI: https://doi.org/10.3399/bjgp18X694409 


\section{How this fits in}

The quality of antimicrobial prescribing in NHS primary care is currently assessed by using item-based weighted prescribing unit rate comparisons. The European Surveillance of Antimicrobial Consumption Network (ESAC-Net) developed and validated disease-specific antibiotic prescribing quality indicators for primary care. The performance of UK primary care against these indicators is unknown. This study provides an overview of the quality of antibiotic prescribing using ESAC- Net quality indicators for young children presenting to primary care and recruited into the Diagnosis of Urinary Tract infection in Young children observational study.

the disease-specific quality of antibiotic prescribing for common infections in young children. This study sought to examine the variations in quality of antibiotic prescribing by factors such as level of deprivation, site type, and demographics. The objectives were to examine the extent to which the ESACNet disease-specific quality indicators are achieved in a population of children aged $\leq 5$ years, at the point of presentation to UK primary care, and to determine predictors for good or poor antibiotic prescribing. Existing data were used, taken from 7163 children aged $<5$ years old, which were collected for the Diagnosis of Urinary Tract infection in Young children (DUTY) study.

\section{METHOD}

\section{Data collection}

The clinical data were originally collected for the DUTY study, a prospective observational, multicentre study that recruited children from primary care in urban and rural areas across England and Wales. A total of 326 sites agreed to participate and 234 of these actively recruited at least one participant. ${ }^{20}$

Parents and children were invited to take part in the study via telephone when booking their appointment or on arrival at the site, where they were given information sheets. If the parent agreed and the child was eligible, written consent was obtained. ${ }^{20}$

Children were included if they were aged between 3 months and 5 years, presenting at a participating site with an acute illness ( $\leq 28$ days) as the predominant concern and with at least one 'constitutional' symptom identified by the National Institute for Health and Care Excellence (NICE) ${ }^{21}$ as a potential marker for urinary tract infections, that is, fever, vomiting, lethargy/malaise, irritability, poor feeding, or failure to thrive. Children $<5$ years were the focus of the DUTY study because younger children are thought to be most at risk from potential complications from urinary tract infections. Children consulting with other 'obvious' causes for their symptoms, such as otitis media, were included as long as the exclusion criteria did not apply. Patients presenting with trauma as a predominant concern were excluded. Full details of eligibility criteria can be found in the DUTY protocol. ${ }^{20}$

Case report forms were completed for all consented patients. These included data on eligibility, registration, presenting symptoms, medical history lincluding recent antibiotic use), clinical examination, and management. Unique identification numbers were sequentially generated and used on consent forms and case report forms. ${ }^{20}$ The diagnosis for the study was the working diagnosis the clinician arrived at before any other tests were undertaken. The subsequent prescription was based on the working diagnosis. Clinicians were made aware of urine dipstick results once they were available, and after they had completed the working diagnosis on the case report form.

\section{Quality assessment standards}

ESAC-Net produced quality indicators for each of the seven main indications for

\section{Table 1. European Surveillance of Antimicrobial Consumption Network disease-specific quality indicators}

used in this study ${ }^{7}$

\begin{tabular}{lllcc} 
Number & Infection & Age range & $\begin{array}{c}\text { Acceptable range } \\
\text { receiving } \\
\text { antibiotics, } \%\end{array}$ & $\begin{array}{c}\text { Acceptable range } \\
\text { receiving recommended } \\
\text { antibiotic, } \%\end{array}$ \\
\hline 1 & Acute URTI & $>1$ year old & $0-20$ & $80-100$ \\
\hline 2 & Acute tonsillitis & $>1$ year old & $0-20$ & $80-100$ \\
\hline 3 & Acute otitis media & $>2$ years old & $0-20$ & $80-100$ \\
\hline URTI = upper respiratory tract infection. & & & $0-5$ \\
\hline
\end{tabular}




\section{Box 1. Antibiotics recommended by the National Institute for Health and Care Excellence ${ }^{21}$

\begin{tabular}{llll} 
When recommended & Acute URTI & Acute tonsillitis & Acute otitis media \\
\hline First-line & Amoxicillin or penicillin V & Penicillin V & Amoxicillin \\
\hline Second-line or penicillin allergy & Clarithromycin & Clarithromycin & Erythromycin \\
\hline
\end{tabular}

antibiotic prescribing. Full details can be found in the ESAC-Net disease-specific quality indicator report. ${ }^{7}$ Quality indicators for three of these indications are applicable to children: acute upper respiratory tract infections (URTIs), acute tonsillitis, and acute otitis media/myringitis (Table 1). ${ }^{7}$ The prescribing data were compared with these.

NICE recommends a 'no antibiotic' or 'delayed antibiotic prescribing' strategy to be adopted for adults and children with an acute URTI, acute otitis media, or tonsillitis. Immediate prescriptions (Box 1) should be given for severe infections or those who are immunocompromised, particularly if sepsis is suspected. ${ }^{21,22}$

\section{Data analysis}

Data were analysed using Stata software (version 13.1).

A descriptive analysis was conducted showing the proportion of children with each disease who were prescribed antibiotics, and the proportion of these children who were

\section{Table 2. Baseline demographics of study participants}

\begin{tabular}{lccc} 
When recommended & $\begin{array}{c}\text { Acute } \\
\text { URTI }\end{array}$ & $\begin{array}{c}\text { Acute } \\
\text { tonsillitis }\end{array}$ & $\begin{array}{c}\text { Acute otitis } \\
\text { media }\end{array}$ \\
\hline Total number of children included & 2268 & 338 & 698 \\
\hline Sex, $n(\%)$ & $1138(50.18)$ & $182(53.85)$ & $353(50.57)$ \\
Male & $1130(49.82)$ & $156(46.15)$ & $345(49.43)$ \\
Female & $2.07(1.37)$ & $3.01(1.27)$ & $2.52(1.31)$ \\
Mean age (SD) & & & \\
\hline Deprivation quintile, $n(\%)$ & 2240 & 336 & 691 \\
Data available & $552(24.64)$ & $63(18.75)$ & $128(18.52)$ \\
1 (Most deprived) & $503(22.46)$ & $79(23.51)$ & $127(18.38)$ \\
2 & $481(21.47)$ & $64(19.05)$ & $130(18.81)$ \\
3 & $358(15.98)$ & $46(13.69)$ & $128(18.52)$ \\
4 & $346(15.45)$ & $84(25.0)$ & $178(25.76)$ \\
5 (Least deprived) & & & \\
\hline Ethnicity, $n(\%)$ & $1803(79.50)$ & $286(84.62)$ & $618(88.54)$ \\
White & $465(20.50)$ & $52(15.38)$ & $80(11.46)$ \\
Non-white & & & \\
\hline Site type, $n(\%)$ & $2195(96.78)$ & $307(90.83)$ & $675(96.70)$ \\
GP practice & $19(0.84)$ & $3(0.89)$ & $8(1.15)$ \\
Walk-in clinic & $54(2.38)$ & $28(8.28)$ & $15(2.15)$ \\
Children's emergency department & & & \\
\hline
\end{tabular}

$S D=$ standard deviation. URTI = upper respiratory tract infection. prescribed the recommended antibiotic. These prescribing data were compared with the ESAC-Net quality indicators to show how well antibiotic prescribing in the UK conforms to the ESAC-Net proposed standards. The appropriateness of the antibiotic prescription was based on Public Health England guidance ${ }^{22}$ as relevant to the clinician's working diagnosis. All included participants had a working diagnosis.

The differences in prescribing rates were examined by level of deprivation, age, sex, ethnicity, and site type. The level of deprivation was calculated using individual patients' postcodes, which were linked to the English and Welsh indices of multiple deprivations, and aggregated from deciles into quintiles. Cuzick's test (non-parametic trend) was used to test for trends across the ordered groups (level of deprivation and age). A $\chi^{2}$ test was performed on the dichotomous variables (sex and ethnicity) and the categorical site type. Trends or differences were considered significant if the $P$-value was $<0.05$.

Both immediate and delayed prescriptions were included in the analysis. Delayed antibiotic prescriptions accounted for $12.92 \%$ of all prescriptions. Acute URTIs included acute sore throat, acute pharyngitis, common cold, acute cough, and acute rhinosinusitis. Doxycycline and quinolones were not included in the results tables because they were not prescribed.

\section{RESULTS}

A total of 7374 children aged $<5$ years were recruited. Of these, 211 were excluded or withdrawn, so 7163 were left with data. A full study flowchart can be found in the previous publication. ${ }^{23}$ Baseline demographics are given in Table 2 for the 3304 in the study, the subsequent 3859 received a working diagnosis other than acute URTI, tonsillitis or otitis media.

\section{Acute URTIs}

Of those children diagnosed with an URTI, $9.48 \%$ were prescribed antibiotics; this conforms to the ESAC-Net standards (0-20\%). However, only $65.11 \%$ of these children were prescribed the recommended, first-line antibiotics (amoxicillin or penicillin V), which is below the acceptable range (Table 3 ). Children from more deprived areas are less likely to be prescribed antibiotics for an URTI $(P=0.038)$. There was a significant increasing trend in prescribing rates as the child's age increased ( $P=0.013)$. White children were more likely to be prescribed antibiotics than children from different ethnic backgrounds $(P=0.049)$. Sex and site 


\section{Table 3. Results compared with European Surveillance of Antimicrobial Consumption Network disease-specific quality indicators}

\begin{tabular}{lccc} 
Variable & URTI & Tonsillitis & Otitis media \\
\hline Receiving antibiotics, \% $(n)$ & $9.48(215 / 2268)$ & $\mathbf{7 1 . 6 0}(242 / 338)$ & $\mathbf{7 0 . 4 9}(492 / 698)$ \\
\hline $95 \% \mathrm{Cl}$ & 8.3 to 10.7 & 66.8 to 76.4 & 67.1 to 73.9 \\
\hline ESAC-Net acceptable range, \% & $0-20$ & $0-20$ & $0-20$ \\
\hline Receiving recommended antibiotic, \% $(\boldsymbol{n})$ & $\mathbf{6 5 . 1 1}(140 / 215)$ & $\mathbf{6 8 . 6 0}(166 / 242)$ & $91.87(452 / 492)$ \\
\hline $95 \% \mathrm{Cl}$ & 58.7 to 72.0 & 62.7 to 74.5 & 89.7 to 94.5 \\
\hline ESAC-Net acceptable range, \% & $80-100$ & $80-100$ & $80-100$ \\
\hline
\end{tabular}

Numbers in bold indicate levels outside the ESAC-Net recommended ranges. ESAC-Net = European

Surveillance of Antimicrobial Consumption Network. URTI = upper respiratory tract infection.

type did not influence prescribing rates and none of the factors influenced the likelihood of receiving the recommended antibiotic (Table 4).

\section{Acute tonsillitis}

The results show that $71.60 \%$ of children diagnosed with tonsillitis were prescribed antibiotics. This exceeds the ESAC-Net quality indicators acceptable range of $0-20 \%$. Of these children, only $68.60 \%$ were prescribed the recommended, first-line antibiotic (penicillin V), which is below the acceptable range (Table 3). Children living in more deprived areas were less likely to be prescribed antibiotics $(P=0.027)$. There was a significant increasing trend in prescribing rates as the child's age increased $(P=0.004)$. Ethnicity, sex, and site type did not influence prescribing rates and none of the factors influenced the likelihood of receiving the recommended antibiotic (Table 4).

\section{Acute otitis media}

Children diagnosed with acute otitis media were prescribed antibiotics $70.49 \%$ of the time. This exceeds the ESACNet quality indicator acceptable range of $0-20 \%$. However, $91.87 \%$ received the recommended first-line antibiotic

Table 4. Potential factors influencing the quality of prescribing

\begin{tabular}{|c|c|c|c|c|c|c|}
\hline \multirow[b]{2}{*}{ Factor } & \multicolumn{2}{|c|}{ URTI } & \multicolumn{2}{|c|}{ Tonsillitis } & \multicolumn{2}{|c|}{ Otitis media } \\
\hline & $\begin{array}{l}\text { Children with } \\
\text { an acute } \\
\text { URTI prescribed } \\
\text { antibiotic, \% }\end{array}$ & $\begin{array}{l}\text { Receiving amoxicillin } \\
\text { or penicillin V, of } \\
\text { those prescribed } \\
\text { an antibiotic, \% }\end{array}$ & $\begin{array}{l}\text { Children with } \\
\text { acute tonsillitis } \\
\text { prescribed } \\
\text { antibiotic, \% }\end{array}$ & $\begin{array}{c}\text { Receiving } \\
\text { penicillin V, of } \\
\text { those prescribed } \\
\text { an antibiotic, } \%\end{array}$ & $\begin{array}{l}\text { Children with } \\
\text { otitis media } \\
\text { prescribed } \\
\text { antibiotic, \% }\end{array}$ & $\begin{array}{c}\text { Receiving } \\
\text { amoxicillin, of } \\
\text { those prescribed } \\
\text { an antibiotic, \% }\end{array}$ \\
\hline \multicolumn{7}{|l|}{ Level of deprivation } \\
\hline 1 (Most deprived) & 9.24 & 60.78 & 68.25 & 58.14 & 68.75 & 92.05 \\
\hline 2 & 6.36 & 65.63 & 65.82 & 71.15 & 60.63 & 90.91 \\
\hline 3 & 10.60 & 66.67 & 70.31 & 68.89 & 70.00 & 94.51 \\
\hline 4 & 10.34 & 72.97 & 67.39 & 70.97 & 67.97 & 87.36 \\
\hline 5 (Least deprived) & 12.14 & 59.52 & 83.33 & 71.43 & 80.34 & 94.41 \\
\hline$P$-value & 0.038 & 0.809 & 0.027 & 0.247 & 0.005 & 0.699 \\
\hline \multicolumn{7}{|l|}{ Age, years } \\
\hline 0 & 8.26 & 63.64 & 62.50 & 86.67 & 75.00 & 94.87 \\
\hline 1 & 8.30 & 68.89 & 59.09 & 69.23 & 65.61 & 91.94 \\
\hline 2 & 9.44 & 56.41 & 72.41 & 59.52 & 77.10 & 89.11 \\
\hline 3 & 10.65 & 70.73 & 74.00 & 68.92 & 67.11 & 93.14 \\
\hline 4 & 13.36 & 65.71 & 80.00 & 69.44 & 71.31 & 91.95 \\
\hline$P$-value ${ }^{a}$ & 0.013 & 0.791 & 0.004 & 0.638 & 0.838 & 0.680 \\
\hline \multicolumn{7}{|l|}{ Ethnic group } \\
\hline White & 28.22 & 65.38 & 73.08 & 68.90 & 73.08 & 68.90 \\
\hline Non-white & 18.06 & 63.64 & 63.46 & 66.67 & 63.46 & 66.67 \\
\hline$P$-value ${ }^{b}$ & 0.049 & 0.847 & 0.158 & 0.798 & 0.096 & 0.595 \\
\hline \multicolumn{7}{|l|}{ Sex } \\
\hline Male & 9.67 & 70.91 & 75.82 & 70.29 & 69.69 & 92.68 \\
\hline Female & 9.29 & 59.05 & 66.67 & 66.35 & 71.30 & 91.46 \\
\hline$P$-value ${ }^{b}$ & 0.761 & 0.069 & 0.063 & 0.514 & 0.640 & 0.617 \\
\hline \multicolumn{7}{|l|}{ Site type } \\
\hline GP surgery & 9.57 & 67.76 & 71.34 & 68.04 & 70.07 & 91.75 \\
\hline Emergency department & 7.41 & 75.00 & 75.00 & 71.43 & 80.00 & 100.00 \\
\hline Walk-in clinic & 5.26 & 100.00 & 66.67 & 100.00 & 87.50 & 100.00 \\
\hline$P$-value ${ }^{b}$ & 0.711 & 0.698 & 0.921 & 0.167 & 0.402 & 0.448 \\
\hline
\end{tabular}

${ }^{a}$ Non-parametric trends. ${ }^{b} X^{2}$ test. URTI $=$ upper respiratory tract infection. 
(amoxicillin), which is within the acceptable range (Table 3). Children from more deprived areas were less likely to be prescribed antibiotics $(P=0.005)$. Age, ethnicity, sex, and site type did not influence prescribing rates and none of the factors influenced the likelihood of receiving the recommended antibiotic (Table 4).

\section{DISCUSSION}

\section{Summary}

The quality of antibiotic prescribing in this dataset was mixed. The prescription rates fall within the recommendations for more general diagnoses such as URTIs, but for specific diagnoses, such as tonsillitis and acute otitis media, the rates exceed the recommended limits.

One of the reasons could be that children with a specific diagnosis might have been more unwell than those with URTIs, for whom many patients would have had nonspecific and milder symptoms. Diagnoses of sore throat or pharyngitis were included in the 'URTI' category rather than with 'tonsillitis', and clinicians may be more likely to diagnose 'sore throat' when they are not going to prescribe antibiotics and diagnose 'tonsillitis' if they are going to prescribe antibiotics. Clinicians might be less confident in not prescribing when the diagnosis is specific and the type of antibiotic to be used is known or clear.

This is also reflected in an acceptable proportion of patients receiving the recommended antibiotic for a specific diagnosis of acute otitis media, but not for URTIs or tonsillitis, where the diagnosis might not be as clear. There are some situations where children require antibiotics but cannot receive the first-line antibiotic for example, if they have a penicillin allergy. About $10 \%$ of the population believe they have a penicillin allergy and treatment with broad-spectrum, non-penicillin antibiotics can lead to antibiotic resistance. ${ }^{24}$ However, this low percentage does not account for the low proportions of children receiving the first-line antibiotic seen in this study.

In the analyses, children from more deprived areas were found to be less likely to be prescribed an antibiotic for all infections. This is an interesting finding, because it could be argued that parents from less deprived areas may have higher health literacy levels, and higher knowledge of antibiotic use, ${ }^{25}$ so are less likely to expect a prescription for self-limiting infections. However, once a decision has been made to consult a GP, many would feel that their child's infection would respond to antibiotics and may pressurise the clinician to prescribe. ${ }^{16}$ Parents with higher literacy levels may understand the threat of antibiotic resistance, but may fail to see how this could affect their child at an individual level and would prefer to err on the side of caution and use antibiotics. Parents from more deprived backgrounds may feel more confident in letting their GPs decide the best course of action.

In general, antibiotic use is higher in more deprived areas, probably as a result of higher incidences of comorbidities and illnesses. ${ }^{26}$ However, this is not reflected in the current study, which only involved children aged $<5$ years and the use of disease-specific prescribing as opposed to the use of item-based prescribing rates for overall population. This may be an example of the inverse care law, with those of higher socioeconomic status being prescribed more antibiotics, in this case potentially leading to greater harm as a result of unnecessary antibiotics.

Prescribing behaviours are complex and influenced by many factors, ${ }^{27}$ therefore, more research is needed to assess how greater knowledge and higher health literacy levels affect prescribing rates. Children with URTIs or tonsillitis were more likely to be prescribed antibiotics as their age increased. This may be because it is easier to diagnose older children. Increased confidence in the diagnosis and choice of antibiotic may increase prescribing rates.

\section{Strengths and limitations}

This is a novel study, analysing the quality of antibiotic prescribing in a large cohort of children presenting in UK primary care by comparison with the ESAC-Net quality indicators and exploring what factors influence prescribing decisions. The study aimed to represent current practice, on the basis of clinicians responses to questions about working diagnoses and testing and treatment plans.

However, the data used were crosssectional, therefore, inferences of causality cannot be made. Additionally, these data were collected in a research environment, which may vary from routine practice. GPs might be more cautious when prescribing antibiotics if they are aware that data are being collected for research. Conversely, the cohort of children may be more unwell than normal practice because they had systemic symptoms, so GPs may have been more inclined to prescribe antibiotics. A sample selection bias may limit the generalisability of the results.

Acute tonsillitis and acute otitis media are often classed as URTIs; however, they were 


\section{Funding}

The Diagnosis of Urinary Tract infection in Young children (DUTY) study was commissioned and funded by the National Institute for Health Research (NIHR) Health Technology Assessment (HTA) programme (project number 08/66/01). Alastair D Hay is funded by NIHR Research Professorship (NIHR-RP-02-12-012). The views and opinions expressed by the authors do not necessarily reflect those of the NHS, NIHR, HTA, or the Department of Health..$^{20}$

\section{Ethical approval}

Multicentre ethical approval was granted by the South West Southmead Research Ethics Committee (Ref \#09/H0102/64). Research and Development (R\&D) approval was granted for all sites recruited..$^{20}$

\section{Provenance}

Freely submitted; externally peer reviewed.

\section{Competing interests}

The authors have declared no competing interests.

\section{Acknowledgements}

Thanks to the children, their families, the general practices, other recruitment sites, and the entire DUTY study team. Thanks also to the providers of nursing/clinical study officer support from the Primary Care Research Networks Greater London; Kent and Medway; Sussex; Surrey; Thames Valley; the Western; Peninsular; Cumbria and Lancashire; Northumberland and Tyne and Wear; and the National Institute for Social Care and Health Research, Clinical Research Centre in Wales INISCHR $\mathrm{CRC}$ ). Additionally, the authors wish to acknowledge the support given by the South East Wales Trials Unit lfunded by NISCHR), the Wales School of Primary Care Research (funded by NISCHR), the Comprehensive Local Research Networks of Central and East London, Western, Peninsula, Hampshire, and Isle of Wight, and the NIHR Biomedical Research and Development Department, Guy's and St Thomas' NHS Foundation Trust.

\section{Discuss this article}

Contribute and read comments about this article: bjgp.org/letters

considered as separate indicators because there is less evidence to support antibiotic prescribing for URTIs than for tonsillitis or otitis media.?

Children aged $<5$ years were included in the analysis despite the age range for the quality indicators being defined as $>1$ or $>2$ years old

Delayed antibiotic prescribing could also be a potential source of bias in interpreting the results; however, it is unlikely to account for the high prescription rates or influence the conclusion because of the small percentage $(12.92 \%)$ of delayed prescriptions. It is also difficult to measure the number of children who actually took the antibiotics that were prescribed. The authors of the ESAC-Net guidelines acknowledge that delayed prescriptions may be a source for potential variation, which may bias the interpretation of their quality indicators.

The analyses presented are descriptive and are not derived from formal hypothesis testing. Therefore, the multiple unadjusted comparisons do not require any correction or adjustment. ${ }^{28}$ However, any association is purely descriptive and should be considered with caution.

\section{Comparison with existing literature}

A UK study, using the Clinical Practice Research Datalink dataset, found that GP practices were overprescribing antibiotics for respiratory tract infections, which is in line with the overall results of the current study, because they included acute otitis media and tonsillitis as respiratory tract infections. ${ }^{9}$ Another study, conducted in Belgium, using the ESAC-Net quality indicators, also found that antibiotics were being overprescribed for common infections in primary care. ${ }^{17} \mathrm{~A}$ French study using ESAC-Net quality indicators found that GPs prescribed $54 \%$ more antibiotics than paediatricians. ${ }^{18}$

In 2012, antibiotic consumption in UK primary care was slightly lower than the European average at 20.1 defined daily dose/1000 inhabitants, compared with 21.5 defined daily dose/1000 for Europe. There were large variations between different countries, suggesting that there is major scope for reducing antibiotic use in the UK and in other European countries. ${ }^{19}$

Another study assessed predictors for antibiotic prescribing in children and found that prescribing rates increased as the age of the child increased and that children were more likely to be prescribed antibiotics if they were diagnosed with tonsillitis or acute otitis media. ${ }^{5}$ These results are also in line with the findings of the current study.

\section{Implications for research and practice}

Despite the publication of clinical guidelines, ${ }^{22}$ this study highlights the need to improve the quality of antibiotic prescribing for children in UK primary care. The outputs of this project provide a benchmark to allow comparison with other European countries and can be used to help target and develop future antibiotic stewardship programmes. However, the results should be viewed with caution as only univariate associations are shown.

The Clinical Practice Research Datalink dataset should be used to determine the standards of antibiotic prescribing for children using the ESAC-Net quality indicators, because it would be more representative of UK antibiotic prescribing than the dataset used in the current study. The quality of antibiotic prescribing in Wales could be assessed using the Secure Anonymised Information Linkage Databank, and then compared with other UK countries.

There is a need to explore the effects of differences in antibiotic prescribing quality on symptom recovery in children. GPs may require more evidence about the harms and benefits of prescribing antibiotics for common infections in children to increase their confidence in non-antibiotic prescribing strategies. Given the increasing emergence of antibiotic resistance and limited development of new, replacement antibiotics, effective professional and public measures should be examined and implemented to encourage appropriate antibiotic prescribing. 


\section{REFERENCES}

1. Goossens H, Ferech M, Vander Stichele R, Elseviers M. Outpatient antibiotic use in Europe and association with resistance: a cross-national database study. Lancet 2005; 365(9459): 579-587.

2. Davies S. Chief Medical Officer annual report 2011: volume 2. 2013. https://www. gov.uk/government/publications/chief-medical-officer-annual-report-volume-2 laccessed 5 Jan 2018).

3. Finch R. Innovation: drugs and diagnostics. J Antimicrob Chemother 2007; 60(Suppl 1): i79-i82.

4. Boucher HW, Talbot GH, Bradley JS, et al. Bad bugs, no drugs: no ESKAPE! An update from the Infectious Diseases Society of America. Clin Infect Dis 2009 48(1): $1-12$

5. O'Brien K, Bellis TW, Kelson M, et al. Clinical predictors of antibiotic prescribing for acutely ill children in primary care: an observational study. Br J Gen Pract 2015; DOl: https://doi.org/10.3399/bjgp15X686497.

6. Butler CC, Hood K, Verheii T, et al. Variation in antibiotic prescribing and its impact on recovery in patients with acute cough in primary care: prospective study in 13 countries. BMJ 2009; 338: b2242.

7. Adriaenssens N, Coenen S, Tonkin-Crine S, et al. European Surveillance of Antimicrobial Consumption (ESAC): disease-specific quality indicators for outpatient antibiotic prescribing. BMJ Qual Saf2011; 20(9): 764-772.

8. Bjerrum L, Munck A, Gahrn-Hansen B, et al. Health Alliance for Prudent Prescribing, Yield and Use of Antimicrobial Drugs in the Treatment of Respiratory Tract Infections (HAPPY AUDIT). BMC Fam Pract 2010; 11(1): 29

9. Gulliford MC, Dregan A, Moore MV, et al. Continued high rates of antibiotic prescribing to adults with respiratory tract infection: survey of $568 \mathrm{UK}$ general practices. BMJ Open 2014; 4(10): e006245.

10. Public Health England. English surveillance programme for antimicrobial utilisation and resistance (ESPAUR) 2010 to 2014. 2015. https://umw.gov.uk/ government/publications/english-surveillance-programme-antimicrobialutilisation-and-resistance-espaur-report (accessed 5 Jan 2018).

11. Costelloe $C$, Metcalfe $C$, Lovering $A$, et al. Effect of antibiotic prescribing in primary care on antimicrobial resistance in individual patients: systematic review and meta-analysis. BMJ 2010; 340: c2096.

12. Paterson DL. The role of antimicrobial management programs in optimizing antibiotic prescribing within hospitals. Clin Infect Dis 2006; 42(Suppl 2): S90-S95.

13. Whaley LE, Businger AC, Dempsey PP, Linder JA. Visit complexity, diagnostic uncertainty, and antibiotic prescribing for acute cough in primary care: a retrospective study. BMC Fam Pract 2013; 14: 120.

14. Kumar S, Little $P$, Britten N. Why do general practitioners prescribe antibiotics for sore throat? Grounded theory interview study. BMJ 2003; 326(7381): 138

15. Lucas PJ, Cabral C, Hay AD, Horwood J. A systematic review of parent and clinician views and perceptions that influence prescribing decisions in relation to acute childhood infections in primary care. Scand J Prim Health Care 2015: 33(1): 11-20.

16. de Bont EG, Peetoom KK, Moser A, et al. Childhood fever: a qualitative study on GPs' experiences during out-of-hours care. Fam Pract 2015; 32(4): 449-455.

17. Adriaenssens H, Bartholomeeusen S, Ryckebosch P, Coenen S. Quality of antibiotic prescription during office hours and out-of-hours in Flemish primary care, using European quality indicators. Eur J Gen Pract 2014; 20(2): 114-120.

18. Pulcini C, Lions $C$, Ventelou B, Verger P. Indicators show differences in antibiotic use between general practitioners and paediatricians. Eur J Clin Microbiol Infect Dis 2013; 32(7): 929-935.

19. Weist K, Muller A, Monnet D, Heuer O. Surveillance of antimicrobial consumption in Europe 2012. Stockholm: European Centre for Disease Prevention and Control, 2014. http://ecdc.europa.eu/en/publications/Publications/antimicrobialconsumption-europe-esac-net-2012.pdf (accessed 5 Jan 2018).

20. Downing $\mathrm{H}$, Thomas-Jones $\mathrm{E}, \mathrm{Gal} \mathrm{M}$, et al. The diagnosis of urinary tract infections in young children (DUTY): protocol for a diagnostic and prospective observational study to derive and validate a clinical algorithm for the diagnosis of UTI in children presenting to primary care with an acute illness. BMC Infect Dis 2012: 12(1): 158.

21. National Institute for Health and Care Excellence. Urinary tract infection in under 16s: diagnosis and management. CG54. London: NICE, 2007. https://umw.nice. org.uk/guidance/cg54 (accessed 5 Jan 2018).

22. McNulty C. Management and treatment of common infections. Antibiotic quidance for primary care: for consultation and local adaption. London: Public Health England, 2017. https://www.gov.uk/government/uploads/system/uploads/ attachment_data/file/455298/230415_Managing_Common_Infections_-_full guide_with_rationale.pdf laccessed 5 Jan 2018).

23. Hay AD, Birnie K, Busby J, et al. The Diagnosis of Urinary Tract infection in Young children (DUTY): a diagnostic prospective observational study to derive and validate a clinical algorithm for the diagnosis of urinary tract infection in children presenting to primary care with an acute illness. Health Technol Assess 2016; 20(51): 1-294.

24. National Institute for Health and Care Excellence. Drug allergy: diagnosis and management. CG183. London: NICE, 2014. https://www.nice.org.uk/guidance/ cg183 (accessed 5 Jan 2018).

25. Nielsen-Bohlman L, Panzer AM, Kindig DA, eds. Health literacy: a prescription to end confusion. Washington, DC: National Academies Press, 2004

26. Wise J. Antibiotic prescribing is higher in deprived areas of England. BMJ 2015; 351: h6117

27. McNulty CA, Boyle P, Nichols T, et al. Don't wear me out: the public's knowledge of and attitudes to antibiotic use. J Antimicrob Chemother 2007; 59(4): 727-738.

28. Savitz DA, Olshan AF. Multiple comparisons and related issues in the interpretation of epidemiologic data. Am J Epidemiol 1995; 142(9): 904-908. 